\title{
Some Remarks on Restricted Panel Data Model
}

Ameera Jaber Mohaisen and Saja Yaseen Abdulsamad

\author{
Mathematics Department College of Education for Pure Science AL-Basrah University-Iraq
}

\begin{abstract}
In this paper, we investigate some remarks on panel data model with linear constraints on the coefficients of the random panel data model. Furthermore, it investigates the inferences. The restricted maximum likelihood method is employed to making inferences on the random panel data model.
\end{abstract}

\section{Keywords}

Panel Data Model; Maximum Likelihood Method; Constrained Model; Restricted Maximum Likelihood Method ; Likelihood Ratio Test

\section{INTRODUCTION}

Regression analysis and time series analysis are two important applied statistical methods used to analyze data. Regression analysis is a special type of multivariate analysis, where several measurements are taken from each subject. We identify one measurement as a response, or dependent variable; the interest is in making statements about this measurement, controlling for the other variables. With regression analysis, it is customary to analyze data from a crosssection of subjects. In contrast, with time series analysis, we identify one or more subjects and observe them over time. This allows us to study relationships over time, the so-called dynamic aspect of a problem. To employ time series methods, we generally restrict ourselves to a limited number of subjects that have many observations over time. Defining longitudinal and panel data Longitudinal data analysis represents a marriage of regression and time series analysis. As with many regression data sets, Iongitudinal data are composed of a cross-section of subjects. Unlike regression data, with longitudinal data we observe subjects over time. Unlike time series data, with longitudinal data we observe many subjects. Observing a broad cross-section of subjects over time allows us to study dynamic, as well as cross-sectional, aspects of a problem. The descriptor panel data comes from surveys of individuals. In this context, a "panel" is a group of individuals surveyed repeatedly over time. Historically, panel data methodology within economics had been largely developed through labor economics applications. Now, economic applications of panel data methods are not confined to survey or labor economics problems and the interpretation of the descriptor "panel analysis" is much broader,[6],[9],[11],[12].

The analysis of panel data allows the model builder to learn about economic processes while accounting for both heterogeneity across individuals, firms, countries, and so on and for dynamic effects that are not visible in cross sections. Modeling in this context often calls for complex stochastic specifications,[13]. The panel data model has been investigated by the many researcher as Elhorst in (2001) presented paper surveys panel data models extended to spatial error autocorrelation or spatially lagged dependent variable, [4]. Hurlin in (2004) proposed a simple test of Granger (1969) non

causality hypothesis in heterogeneous panel data models with fixed coefficients,[8] . Bun , a. e. in (2005) studied extends earlier results on bias - corrected estimators for the fixed effects dynamic panel data model,[3]. Gorgen a. e. in (2008) discussed efficient estimation of nonlinear dynamic panel data models with application to smooth transition models ,they explores estimation of a class of nonlinear dynamic panel data models with additive unobserved individual specific effects,[7]. Feng a. e. in (2015) proposed a panel data Semiparametric varying coefficient model in which covariates (variables affecting the coefficients) are purely categorical,[5]. Ashley and Sun in (2016) proposed subset continuous updating GMM estimators for dynamic panel data models,[1],[2]

Constrained parameter problems arise in a wide variety of applications, including bioassay, actuarial graduation, ordinal data, response surfaces, reliability development testing and variance component model. The normal linear regression model subject to linear inequality constraints for the coefficients arises commonly in applied econometrics as well as other scientific applications. Typically the motivating economic model restricts the sings of certain coefficients or of known linear combinations of coefficients,[10],[11].

The aim of this paper is to deal with restricted panel data model, we investigate some remarks on panel data model with linear constraints on the coefficients of the model. Furthermore, it investigates the inferences on the model. We explore the estimation of restricted panel data model, likelihood ratio test between restricted and unrestricted models, and prove some properties about the parameters estimation.

\section{2- Panel Data Model}

Consider the model:
$\mathrm{Y}_{\mathrm{it}}=\mu+\sum_{\mathrm{j}=1}^{\mathrm{K}} \beta_{\mathrm{j}} \mathrm{X}_{\mathrm{jit}}+\varepsilon_{\mathrm{it},} \mathrm{i}=1_{w \ldots s} \mathrm{~N}_{s} \mathrm{t}=1_{v \infty \ldots} \mathrm{T}_{v}$

where, $Y_{\mathrm{it}}$ the value of response variable for $i^{\mathrm{th}}$ unit at time $\mathrm{t}, \mathrm{X}_{\mathrm{jit}}$ the explanatory variables, $\mu_{x} \beta_{\tilde{j}_{j}} \mathrm{j}=1_{x, x_{x}} \mathrm{~K}$ are fixed parameters and $\varepsilon_{\mathrm{it}}$ is an error term with $\varepsilon_{\mathrm{it}}{ }_{\alpha} \mathrm{Ni}\left(\mathrm{N}_{z} \sigma_{\mathrm{g}}^{2}\right)$.

Now, if the parameter $\mu$ is specified as :

7168 | $\mathrm{P}$ a g e

A pril, 2017 
$\mu=\beta_{0}+u_{i}$

where, $u_{\mathrm{i}} \sim N\left(0_{i}, \sigma_{u}^{2}\right)$, then, the model (1) is

$\mathrm{Y}_{\mathrm{it}}=\beta_{\mathrm{o}}+\sum_{\mathrm{j}=1}^{\mathrm{k}} \beta_{\mathrm{j}} \mathrm{x}_{\mathrm{jit}}+\mathrm{u}_{\mathrm{i}}+\varepsilon_{\mathrm{it}}$.

The model (3) is rewrite as follows :

$\mathrm{Y}_{\mathrm{it}}=\beta_{0}+\sum_{\mathrm{j}=1}^{\mathrm{k}} \beta_{\mathrm{j}} \mathrm{x}_{\mathrm{jit}}+\omega_{\mathrm{it}}$ 。

where, $\omega_{i t}=u_{i}+\varepsilon_{i t}, \omega_{i t} \sim N\left(0, \sigma_{\omega}^{2}\right), \sigma_{\omega}^{2}=\sigma_{\varepsilon}^{2}+\sigma_{u}^{2}$, thus by using matrix notation the model (4) is $\mathrm{Y}=\mathrm{F} \theta+\mathrm{w}_{\mathrm{o}}$

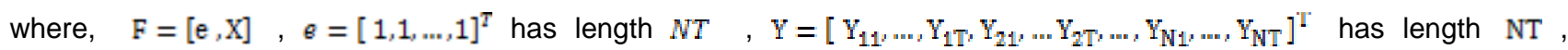
$\mathrm{X}=\left[\mathrm{X}_{1,}, \mathrm{X}_{2^{* \ldots x},} \mathrm{X}_{\mathrm{N}}\right]^{\mathrm{T}}$ is a $N T \times K$ design matrix of fixed effects, $\theta=\left[\beta_{00}, \beta_{1, \ldots x}, \beta_{\mathrm{K}}\right]^{\mathrm{T}}$ has length $K+1$, ands

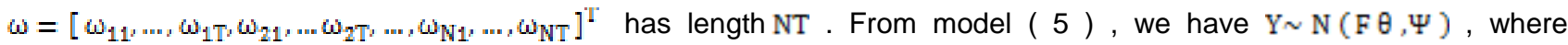
$\Psi=\mathrm{E}\left(\omega \omega^{\mathrm{T}}\right)=\mathrm{I}_{\mathrm{N}} \otimes\left(\sigma_{\mathrm{z}}^{2} \mathrm{I}_{\mathrm{t}}+\sigma_{\mathrm{u}}^{2} \mathrm{ee}{ }^{\mathrm{I}}\right)$

$$
=\sigma_{\mathrm{z}}^{2}\left(\mathrm{I}_{\mathrm{N}} \otimes \mathrm{I}_{\mathrm{t}}\right)+\sigma_{\mathrm{u}}^{2}\left(\mathrm{I}_{\mathrm{N}} \otimes \mathrm{ee}^{\mathrm{I}}\right),
$$

replace $\mathrm{I}_{\mathrm{T}}$ by $\left(\mathrm{E}_{\mathrm{T}}+\mathrm{J}_{\mathrm{T}}\right)$ and $e \mathrm{e}^{\mathrm{T}}$ by $\mathrm{T} \mathrm{J}_{\mathrm{T}}$, where $\mathrm{I}_{\mathrm{T}}=\frac{1}{T} e e^{T}$ and $\mathrm{E}_{\mathrm{T}}=\mathrm{I}_{\mathrm{T}}-\mathrm{J}_{\mathrm{T}}$, then

$\Psi=\sigma_{\mathrm{z}}^{2}\left[\mathrm{I}_{\mathrm{N}} \otimes\left(\mathrm{E}_{\mathrm{T}}+\mathrm{J}_{\mathrm{T}}\right)\right]+\sigma_{\mathrm{u}}^{2}\left(\mathrm{I}_{\mathrm{N}} \otimes \mathrm{T} \mathrm{J}_{\mathrm{T}}\right)$

$=\sigma_{\mathrm{g}}^{2}\left(\mathrm{I}_{\mathrm{N}} \otimes \mathrm{E}_{\mathrm{T}}\right)+\sigma_{\mathrm{g}}^{2}\left(\mathrm{I}_{\mathrm{N}} \otimes \mathrm{J}_{\mathrm{T}}\right)+\mathrm{T} \sigma_{\mathrm{u}}^{2}\left(\mathrm{I}_{\mathrm{N}} \otimes \mathrm{J}_{\mathrm{T}}\right)$,

by collecting terms with the same matrices, we get

$\Psi=\sigma_{\mathrm{g}}^{2}\left(\mathrm{I}_{\mathrm{N}} \otimes \mathrm{E}_{\mathrm{T}}\right)+\left(\sigma_{\mathrm{g}}^{2}+\mathrm{T} \sigma_{\mathrm{u}}^{2}\right)\left(\mathrm{I}_{\mathrm{N}} \odot \mathrm{J}_{\mathrm{T}}\right)=\sigma_{\mathrm{g}}^{2} \mathrm{Q}+\sigma_{1}^{2} \mathrm{P}$, where,$\sigma_{1}^{2}=\left(\sigma_{\mathrm{g}}^{2}+\mathrm{T} \sigma_{u}^{2}\right)$ and $\Psi^{-1}=\frac{\mathrm{Q}}{\sigma_{\mathrm{z}}^{2}}+\frac{\mathrm{P}}{\sigma_{1}^{2}}$,

$|\Psi|=$ product of its characteristic roots, $[2] \rightarrow|\Psi|=\left(\sigma_{g}^{2}\right)^{\mathrm{N}(\mathrm{T}-1)}\left(\sigma_{1}^{2}\right)^{\mathrm{N}}$.

Since $Y \sim \mathrm{N}_{\mathrm{NT}}(F \theta, \Psi)$, then, the density function of $Y$ is

$f\left(Y_{0}, \theta_{0} \Psi\right)=(2 \pi)^{\frac{-N T}{2}}|\Psi|^{\frac{-1}{2}}$ exp $\left\{\frac{-1}{2}(Y-F \theta)^{\mathbb{T}} \Psi^{-1}(Y-F \theta)\right\}$, then, the likelihood function is the joint density of the $Y^{s} S$ that is

$\mathrm{L}\left(\mathrm{Y} ; \theta_{x} \Psi\right)=(2 \pi)^{\frac{-W T}{2}}|\Psi|^{\frac{-2}{2}} \exp \left\{\frac{-1}{2}(\mathrm{Y}-\mathrm{F} \theta)^{\mathrm{T}} \Psi^{-1}(\mathrm{Y}-\mathrm{F} \theta)\right\}$

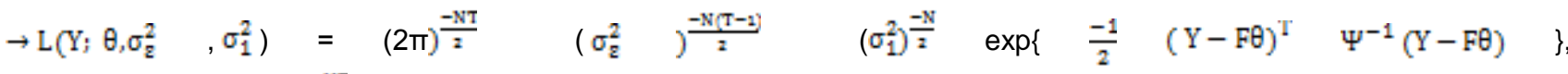

$\therefore L$

$$
\left(\sigma_{1}^{2}\right)^{\frac{-N}{2}} \exp \left\{\frac{-1}{2}(Y-F \theta)^{T}\left[\frac{Q}{\sigma_{2}^{2}}+\frac{P}{\sigma_{2}^{2}}\right](Y-F \theta)\right\} \text {. }
$$

$\rightarrow L n L=\frac{-N T}{2} \ln (2 \pi)-\frac{N(T-1)}{2} \ln \sigma_{\mathrm{g}}^{2}-\frac{N}{2} \ln \sigma_{1}^{2}\left\{\frac{-1}{2}(Y-F \theta)^{T}\left[\frac{\mathrm{Q}}{\sigma_{2}^{2}}+\frac{\mathrm{p}}{\sigma_{2}^{2}}\right](\mathrm{Y}-\mathrm{F} \theta)\right\}$.

Then

$\hat{\theta}=\left(F^{T} \Psi^{-1} F\right)^{-1}\left(F^{T} \Psi^{-1} Y\right)$.

$\hat{\sigma}_{z}^{2}=\frac{1}{\mathrm{~N}(\mathrm{~T}-1)}(\mathrm{Y}-\mathrm{F} \hat{\theta})^{\mathrm{T}} \mathrm{Q}(\mathrm{Y}-\mathrm{F} \hat{\theta})$.

$\hat{\sigma}_{1}^{2}=\frac{1}{\mathrm{~N}}(\mathrm{Y}-\mathrm{F} \hat{\theta})^{\mathrm{T}} \mathrm{P}(\mathrm{Y}-\mathrm{F} \hat{\theta})$.

\section{3- Constrained maximum likelihood estimator}

In this section we consider a set of $\mathrm{m}$ linear constraints on the coefficients of the random panel data model (5).Furthermore, it investigates the inferences. The restricted maximum likelihood method is employed to making inferences on the random panel data model.

Consider the model (5) above, we assume that

$R \theta=r_{x}$ 
where $R$ is $m \times K^{*}, \mathrm{r}$ is $m \times 1, \theta$ is $K^{*} \times 1$ and $\mathrm{K}^{*}=\mathrm{K}+1$.

Then, the restricted likelihood is

$\mathrm{L}\left(\mathrm{Y}_{*}, \theta_{2}, \sigma_{\mathrm{g}}^{2}, \sigma_{1}^{2}\right)=(2 \pi)^{\frac{-\mathrm{NT}}{2}}\left(\sigma_{\mathrm{g}}^{2}\right)^{\frac{-\mathrm{N}[\mathrm{T}-2 \mathrm{~s}]}{2}}$

$$
\left(\sigma_{1}^{2}\right)^{\frac{-N}{2}} \exp \left\{\frac{-1}{2}\left(Y-F \theta^{C}\right)^{T}\left[\frac{Q}{\sigma_{2}^{2}}+\frac{\mathrm{P}}{\sigma_{1}^{2}}\right]\left(Y-F \theta^{C}\right)\right\}
$$

where $\theta^{\mathrm{c}}$ is $\left(K^{*} \times 1\right)$ the restricted vector of parameters, then

$$
\begin{aligned}
& L n L=\frac{-N T}{2} \ln (2 \pi)-\frac{N(T-1)}{2} \ln \sigma_{g}^{2}-\frac{N}{2} \ln \sigma_{1}^{2}\left\{\frac{-1}{2}\left(Y-F \theta^{C}\right)^{T}\left[\frac{Q}{\sigma_{2}^{2}}+\frac{P}{\sigma_{1}^{2}}\right]\left(Y-F \theta^{C}\right)\right\} . \\
& \rightarrow \frac{\partial \operatorname{InL}}{\partial \sigma_{2}^{2}}=-\frac{\mathrm{N}(\mathrm{T}-1)}{2 \sigma_{2}^{2}}+\frac{1}{2}\left(\frac{1}{\sigma_{2}^{2}}\right)^{2}\left(\mathrm{Y}-\mathrm{F} \theta^{\mathrm{C}}\right)^{\mathrm{T}} \mathrm{Q}\left(\mathrm{Y}-\mathrm{F} \theta^{\mathrm{C}}\right)=0 \\
& \rightarrow \frac{\mathrm{N}(\mathrm{T}-1)}{2 \sigma_{2}^{2}}+\frac{1}{2 \sigma_{2}^{4}}\left(\mathrm{Y}-\mathrm{F} \theta^{\mathrm{C}}\right)^{\mathrm{T}} \mathrm{Q}\left(\mathrm{Y}-\mathrm{F}^{\mathrm{C}}\right)=0 \rightarrow \frac{\partial \mathrm{InL}}{2 \sigma_{2}^{2}}=\frac{-\mathrm{N}(\mathrm{T}-1) \sigma_{2}^{2}+(\mathrm{Y}-\mathrm{F \theta})^{\mathrm{T}} \mathrm{Q}(\mathrm{X}-\mathrm{F \theta} 9)}{2 \sigma_{2}^{4}}=0 \\
& \rightarrow \mathrm{N}(\mathrm{T}-1) \hat{\sigma}_{\mathrm{z}}^{2}=\left(\mathrm{Y}-\mathrm{F} \theta \mathrm{C}^{\mathrm{T}} \mathrm{Q}\left(\mathrm{Y}-\mathrm{F} \theta^{\mathrm{C}}\right)\right. \\
& \therefore \hat{\sigma}_{z}^{2}=\frac{1}{\mathbb{N}(\mathrm{T}-1)}\left(\mathrm{Y}-\mathrm{F}^{\mathrm{C}}\right)^{\mathrm{T}} \mathrm{Q}\left(\mathrm{Y}-\mathrm{F} \theta^{\mathrm{C}}\right) \text {. }
\end{aligned}
$$

By the same way we can find that

$\hat{\sigma}_{1}^{2}=\frac{1}{\mathrm{~N}}\left(\mathrm{Y}-\mathrm{F} \theta^{\mathrm{C}}\right)^{\mathrm{T}} \mathrm{P}\left(\mathrm{Y}-\mathrm{F} \theta^{\mathrm{C}}\right)$,

now, by substitute (13), (14) in (12) get

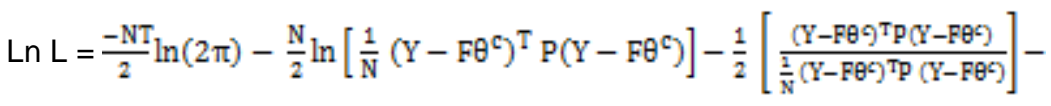

$$
\begin{aligned}
& \frac{\mathrm{N}(\mathrm{T}-1)}{2} \ln \left[\frac{1}{\mathrm{~N}(\mathrm{~T}-1)}\left(\mathrm{Y}-\mathrm{F}^{\mathrm{C}}\right)^{\mathrm{T}} \mathrm{Q}\left(\mathrm{Y}-\mathrm{F}^{\mathrm{C}}\right)\right]-\frac{1}{2}\left[\frac{(\mathrm{Y}-\mathrm{F \theta})^{\mathrm{T}} \mathrm{Q}(\mathrm{Y}-\mathrm{F \theta})}{\frac{1}{\mathrm{~N}(\mathrm{~T}-1)}\left(\mathrm{Y}-\mathrm{F} \theta^{\mathrm{C}}\right)^{\mathrm{T}} \mathrm{Q}\left(\mathrm{Y}-\mathrm{F} \mathrm{F}^{\mathrm{C}}\right)}\right] \text {. }
\end{aligned}
$$

Thus, the restricted likelihood function is given by

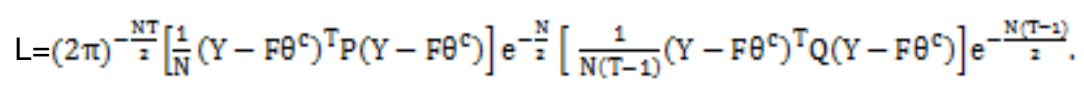

Then ,we can rewrite the description of the problem as the following

$$
\begin{aligned}
\max _{\theta^{e}} \ln L= & \frac{-\mathrm{NI}}{2} \ln (2 \pi)-\frac{\mathrm{N}}{2} \ln \left[\frac{1}{\mathrm{~N}}\left(\mathrm{Y}-\mathrm{F} \theta^{\mathrm{C}}\right)^{\mathrm{T}} \mathrm{P}\left(\mathrm{Y}-\mathrm{F} \theta^{\mathrm{C}}\right)\right]-\frac{\mathrm{N}}{2} \\
& -\frac{\mathrm{N}(\mathrm{T}-1)}{2} \ln \left[\frac{1}{\mathrm{~N}(\mathrm{~T}-1)}\left(\mathrm{Y}-\mathrm{F} \theta^{\mathrm{C}}\right)^{\mathrm{T}} \mathrm{Q}\left(\mathrm{Y}-\mathrm{F} \theta^{\mathrm{C}}\right)\right]-\frac{\mathrm{N}(\mathrm{T}-1)}{2} 。
\end{aligned}
$$

s.t $R \theta^{C}=r$.

Then ,we can maximize this likelihood function (17) by minimizing the terms $\left(\mathrm{Y}-\mathrm{F}^{\mathrm{C}}\right)^{\mathrm{I}} \mathrm{P}\left(\mathrm{Y}-\mathrm{F}^{\mathrm{C}}\right)$ and $\left(\mathrm{Y}-\mathrm{F} \theta^{\mathrm{C}}\right)^{\mathrm{I}} \mathrm{Q}\left(\mathrm{Y}-\mathrm{F \theta}^{\mathrm{C}}\right)$. To satisfy (17) subject to constraint (18), we use the form of Lagrangian function where $\lambda^{\mathrm{T}}$ is $(m \times 1)$ vector of Lagrangian multipliers, thus, we can get

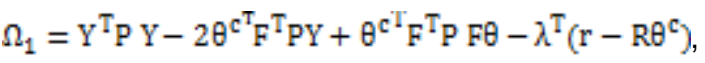

$$
\begin{aligned}
& \Omega_{2}=\mathrm{Y}^{\mathrm{T}} \mathrm{QY}-2 \theta^{\mathrm{c}^{\mathrm{T}}} \mathrm{F}^{\mathrm{T}} \mathrm{QY}+\theta^{\mathrm{C}^{\mathrm{I}}} \mathrm{F}^{\mathrm{T}} \mathrm{P} \mathrm{Q} \mathrm{F} \theta-\lambda^{\mathrm{T}}\left(\mathrm{r}-\mathrm{R} \theta^{\mathrm{C}}\right) \text {, } \\
& \rightarrow \Omega_{1}+\Omega_{2}=\mathrm{Y}^{\mathrm{T}} \mathrm{Y}-2 \theta^{\mathrm{C}^{\mathrm{I}}} \mathrm{F}^{\mathrm{T}} \mathrm{Y}+\theta^{\mathrm{C}^{\mathrm{I}}} \mathrm{F}^{\mathrm{T}} \mathrm{F} \theta-2 \lambda^{\mathrm{T}}\left(\mathrm{r}-\mathrm{R} \theta^{\mathrm{C}}\right) \text { 。 }
\end{aligned}
$$

where $Q+P=I_{N I}$, now, differentiation (19) above with respect to $\theta^{C^{I}}$ and $\lambda$ so fulfilled conditions

$$
\left.\begin{array}{rl}
-2 F^{\mathrm{T}} \mathrm{Y}+2 \mathrm{~F}^{\mathrm{T}} \mathrm{F} \theta^{\mathrm{C}}+2 \lambda \mathrm{R}^{\mathrm{T}} & =0 \\
2\left(\mathrm{R} \theta^{\mathrm{C}}-\mathrm{r}\right) & =0
\end{array}\right\}
$$

Then , by multiply the first equation in (20) by $R\left(\mathrm{~F}^{\mathrm{I}} \mathrm{F}\right)^{-1}$, get

$$
\begin{aligned}
& -2 R\left(F^{\mathbb{I}} F\right)^{-1} F^{\mathbb{I}} Y+2 R\left(F^{\mathbb{I}} F\right)^{-1} F^{\mathbb{I}} F \theta^{C}+2 R\left(F^{\mathbb{I}} F\right)^{-1} \lambda R^{\mathbb{I}}=0 \\
& \rightarrow-2 R\left(F^{\mathbb{I}} \Psi^{-1} F\right)^{-1}\left(F^{\mathbb{I}} \Psi^{-1} Y\right)+2 R \theta^{c}+2 R\left(F^{\mathbb{I}} F\right)^{-1} \lambda R^{\mathbb{I}}=0,
\end{aligned}
$$


since $\hat{\theta}=\left(F^{T} \Psi^{-1} F\right)^{-1}\left(F^{T} \Psi^{-1} Y\right)$. Then (21) is $-2 R \hat{\theta}+2 R \theta^{C}+2 R\left(F^{T} F\right)^{-1} \lambda R^{T}=0$

$\rightarrow\left[R\left(F^{T} F\right)^{-1} R^{T}\right] \lambda=R \hat{\theta}-R \theta^{C}$

$\therefore \lambda=-\left[R\left(F^{T} F\right)^{-1} R^{T}\right]^{-1}(r-R \hat{\theta}), R \theta^{C}=r$.

Now, substitute (22) in the first equation of (20) to get

$-2 F^{\mathrm{I}} \mathrm{Y}+2 \mathrm{~F}^{\mathrm{I}} \mathrm{F}^{\mathrm{C}}+2 \mathrm{R}^{\mathrm{I}} \mathrm{X}=0 \rightarrow-\mathrm{F}^{\mathrm{I}} \mathrm{Y}+\mathrm{F}^{\mathrm{I}} \mathrm{F} \theta^{\mathrm{C}}+\mathrm{R}^{\mathrm{I}} \lambda=0$

$\rightarrow-F^{\mathrm{T}} \mathrm{Y}+\mathrm{F}^{\mathrm{T}} \mathrm{F} \theta^{\mathrm{C}}-\mathrm{R}^{\mathrm{T}}\left(\mathrm{R}\left(\mathrm{F}^{\mathrm{T}} \mathrm{F}\right)^{-1} \mathrm{R}^{\mathrm{T}}\right)^{-1}(\mathrm{r}-\mathrm{R} \hat{\theta})=0$

$\rightarrow \mathrm{F}^{\mathrm{T}} \mathrm{F} \theta^{\mathrm{C}}=\mathrm{F}^{\mathrm{T}} \mathrm{Y}+\mathrm{R}^{\mathrm{T}}\left(\mathrm{R}\left(\mathrm{F}^{\mathrm{T}} \mathrm{F}\right)^{-1} \mathrm{R}^{\mathrm{T}}\right)^{-1}(\mathrm{r}-\mathrm{R} \hat{\theta})$

$\rightarrow \theta^{\mathrm{C}}=\left(\mathrm{F}^{\mathrm{T}} \mathrm{F}\right)^{-1} \mathrm{~F}^{\mathrm{T}} \mathrm{Y}+\left(\mathrm{F}^{\mathrm{T}} \mathrm{F}\right)^{-1} R^{\mathrm{T}}\left(\mathrm{R}\left(\mathrm{F}^{\mathrm{T}} \mathrm{F}\right)^{-1} R^{\mathrm{T}}\right)^{-1}(\mathrm{r}-\mathrm{R} \hat{\theta})$

$\rightarrow \theta^{\mathrm{C}}=\left(\mathrm{F}^{\mathrm{T}} \Psi^{-1} \mathrm{~F}\right)^{-1} F^{\mathrm{T}} \Psi^{-1} Y+\left(\mathrm{F}^{\mathrm{T}} \mathrm{F}\right)^{-1} R^{\mathrm{T}}\left(R\left(\mathrm{~F}^{\mathrm{T}} \mathrm{F}\right)^{-1} R^{\mathrm{T}}\right)^{-1}(\mathrm{r}-\mathrm{R} \hat{\theta})$

$\therefore \theta^{\mathrm{C}}=\hat{\theta}+\left(\mathrm{F}^{\mathrm{T}} \mathrm{F}\right)^{-1} \mathrm{R}^{\mathrm{T}}\left(\mathrm{R}\left(\mathrm{F}^{\mathrm{T}} \mathrm{F}\right)^{-1} \mathrm{R}^{\mathrm{T}}\right)^{-1}(\mathrm{r}-\mathrm{R} \hat{\theta})$.

Therefore,

$\theta^{\mathrm{C}}-\hat{\theta}=\left(\mathrm{F}^{\mathrm{T}} \mathrm{F}\right)^{-1} \mathrm{R}^{\mathrm{T}}\left(\mathrm{R}\left(\mathrm{F}^{\mathrm{T}} \mathrm{F}\right)^{-1} \mathrm{R}^{\mathrm{T}}\right)^{-1}(\mathrm{r}-\mathrm{R} \hat{\theta})$.

$\rightarrow\left(F^{T} F\right)\left(\theta^{\mathrm{C}}-\hat{\theta}\right)=R^{\mathrm{T}}\left(R\left(\mathrm{~F}^{\mathrm{T}} \mathrm{F}\right)^{-1} \mathrm{R}^{\mathrm{T}}\right)^{-1}(\mathrm{r}-\mathrm{R} \hat{\theta})$ 。

$\rightarrow\left(\theta^{\mathrm{C}}-\hat{\theta}\right)^{\mathrm{T}}\left(\mathrm{F}^{\mathrm{T}} \mathrm{F}\right)\left(\theta^{\mathrm{C}}-\hat{\theta}\right)=(\mathrm{r}-\mathrm{R} \hat{\theta})^{\mathrm{T}}\left(\mathrm{R}\left(\mathrm{F}^{\mathrm{T}} \mathrm{F}\right)^{-1} \mathrm{R}^{\mathrm{T}}\right)^{-1} \mathrm{R}\left(\mathrm{F}^{\mathrm{T}} \mathrm{F}\right)^{-1} \mathrm{R}^{\mathrm{T}}\left(\mathrm{R}\left(\mathrm{F}^{\mathrm{T}} \mathrm{F}\right)^{-1} \mathrm{R}^{\mathrm{T}}\right)^{-1}(\mathrm{r}-\mathrm{R} \hat{\theta})$.

We can rewrite (23) by multiply by $F_{x}$ obtain

$$
\begin{aligned}
& \mathrm{F} \theta^{\mathrm{C}}=\mathrm{F} \hat{\theta}+\mathrm{F}\left(\mathrm{F}^{\mathrm{T}} \mathrm{F}\right)^{-1} R^{\mathrm{T}}\left(R\left(\mathrm{~F}^{\mathrm{T}} \mathrm{F}\right)^{-1} R^{\mathrm{T}}\right)^{-1}(\mathrm{r}-\mathrm{R} \hat{\theta}) \\
& \rightarrow \mathrm{Y}-\mathrm{F} \theta^{\mathrm{C}}=\mathrm{Y}-\mathrm{F} \hat{\theta}-\mathrm{F}\left(\mathrm{F}^{\mathrm{T}} \mathrm{F}\right)^{-1} \mathrm{R}^{\mathrm{T}}\left(\mathrm{R}\left(\mathrm{F}^{\mathrm{T}} \mathrm{F}\right)^{-1} \mathrm{R}^{\mathrm{T}}\right)^{-1}(\mathrm{r}-\mathrm{R} \hat{\theta}) \\
& \rightarrow \omega^{\mathrm{C}}=\widehat{\omega}-\mathrm{F}\left(\mathrm{F}^{\mathrm{T}} \mathrm{F}\right)^{-1} R^{\mathrm{T}}\left(R\left(\mathrm{~F}^{\mathrm{T}} \mathrm{F}\right)^{-1} R^{\mathrm{T}}\right)^{-1}(\mathrm{r}-\mathrm{R} \hat{\theta}) .
\end{aligned}
$$

Where $\omega^{C}$ is the estimator residual from the constrained model .

$$
\rightarrow \omega^{c} \omega^{e^{T}}=\left[\hat{\omega}^{\mathrm{T}}-(\mathrm{r}-\mathrm{R} \hat{\theta})^{\mathrm{T}}\left(\mathrm{R}\left(\mathrm{F}^{\mathrm{T}} \mathrm{F}\right)^{-1} \mathrm{R}^{\mathrm{T}}\right)^{-1} \mathrm{R}\left(\mathrm{F}^{\mathrm{T}} \mathrm{F}\right)^{-1} \mathrm{~F}^{\mathrm{T}}\right]
$$

$\left[\hat{\omega}-F\left(F^{T} F\right)^{-1} R^{T}\left(R\left(F^{T} F\right)^{-1} R^{T}\right)^{-1}(r-R \hat{\theta})\right]$, where $\omega^{c} \omega^{e^{T}}$ sum of squared errors from the constrained model.

$$
\begin{aligned}
= & \widehat{\omega}^{T} \hat{\omega}-\widehat{\omega}^{T} F\left(F^{T} F\right)^{-1} R^{T}\left(R\left(F^{T} F\right)^{-1} R^{T}\right)^{-1}(r-R \hat{\theta}) \\
& -(r-R \hat{\theta})^{T}\left(R\left(F^{T} F\right)^{-1} R^{T}\right)^{-1} R\left(F^{T} F\right)^{-1} F^{T} \hat{\omega}+(r-R \hat{\theta})^{T} \\
& \times\left\{\left(R\left(F^{T} F\right)^{-1} R^{T}\right)^{-1} R\left(F^{T} F\right)^{-1} F^{T} F\left(F^{T} F\right)^{-1} R^{T}\right\}\left(R\left(F^{T} F\right)^{-1} R^{T}\right)^{-1}(r-R \hat{\theta}),
\end{aligned}
$$

where $\widehat{\omega} \tilde{\omega}$ is the estimated residual from unconstrained model

$$
\begin{aligned}
& \widehat{\omega}^{T} \hat{\omega}=(\mathrm{Y}-\mathrm{F} \hat{\theta})^{\mathrm{T}}(\mathrm{Y}-\mathrm{F} \hat{\theta})=\mathrm{Y}^{\mathrm{T}} \mathrm{Y}-2 \hat{\theta}^{\mathrm{T}} \mathrm{F}^{\mathrm{T}} \mathrm{Y}+\hat{\theta}^{\mathrm{T}} \mathrm{F}^{\mathrm{T}} \mathrm{F} \hat{\theta} . \\
& \text { where } F^{\mathrm{T}} \hat{\mathrm{\omega}}=\mathrm{F}^{\mathrm{T}}(\mathrm{Y}-\mathrm{F} \hat{\theta})=\mathrm{F}^{\mathrm{T}}\left(\mathrm{Y}-\mathrm{F}\left[\left(\mathrm{F}^{\mathrm{T}} \Psi^{-1} \mathrm{~F}\right)^{-1}\left(\mathrm{~F}^{\mathrm{T}} \Psi^{-1} \mathrm{Y}\right)\right]\right) \\
& =\mathrm{F}^{\mathrm{I}} \mathrm{Y}-\left[\left(\mathrm{F}^{\mathrm{T}} \mathrm{F}\right)\left(\mathrm{F}^{\mathrm{T}} \mathrm{F}\right)^{-1} \mathrm{~F}^{\mathrm{I}} \mathrm{Y}\right. \\
& =\mathrm{F}^{\mathrm{I}} \mathrm{Y}-\mathrm{F}^{\mathbb{1}} \mathrm{Y}=0 \text { 。 }
\end{aligned}
$$

Using this information in equation (22) and (24) to obtain

$$
\begin{aligned}
& \omega^{c} \omega^{\omega^{T}}=\widehat{\omega}^{T} \hat{\omega}-\widehat{\omega}^{T} F\left(F^{T} F\right)^{-1} R^{T}\left(R\left(F^{T} F\right)^{-1} R^{T}\right)^{-1}(r-R \hat{\theta}) \\
& \quad-(r-R \hat{\theta})^{T}\left(R\left(F^{T} F\right)^{-1} R^{T}\right)^{-1} R\left(F^{T} F\right)^{-1} F^{T} \hat{\omega}+(r-R \hat{\theta})^{T}\left(R\left(F^{T} F\right)^{-1} R^{T}\right)^{-1}(r-R \hat{\theta}), \\
& \rightarrow \omega^{c} \omega^{c^{T}}=\widehat{\omega}^{T} \hat{\omega}+(r-R \hat{\theta})^{T}\left(R\left(F^{T} F\right)^{-1} R^{T}\right)^{-1}(r-R \hat{\theta}) \\
& \rightarrow \omega^{c} \omega^{\omega^{T}}-\widehat{\omega}^{T} \hat{\omega}=(r-R \hat{\theta})^{T}\left(R\left(F^{T} F\right)^{-1} R^{T}\right)^{-1}(r-R \hat{\theta}) .
\end{aligned}
$$

Now, by rewrite (23) as following

$\hat{\theta}=\left(\mathrm{F}^{\mathrm{T}} \Psi^{-1} \mathrm{~F}\right)^{-1}\left(\mathrm{~F}^{\mathrm{T}} \Psi^{-1} \mathrm{Y}\right)=\left(\mathrm{F}^{\mathrm{T}} \Psi^{-1} \mathrm{~F}\right)^{-1} \mathrm{~F}^{\mathrm{T}} \Psi^{-1}(\mathrm{~F} \theta+\omega)$ 


$$
\rightarrow \hat{\theta}=\theta+\left(F^{T_{\Psi}-1} F\right)^{-1}\left(F^{T} \Psi^{-1} \omega\right)
$$

then, substitute (26) in (23) to obtain

$$
\begin{aligned}
& \theta^{C}=\hat{\theta}+\left(F^{T} F\right)^{-1} R^{T}\left(R\left(F^{T} F\right)^{-1} R^{T}\right)^{-1}(r-R \hat{\theta}) \\
& =\theta+\left(F^{\mathbb{I}} \Psi^{-1} F\right)^{-1}\left(F^{\mathbb{I}} \Psi^{-1} \omega\right)+\left(F^{\mathbb{I}} F\right)^{-1} R^{\mathbb{I}}\left(R\left(F^{\mathbb{I}} F\right)^{-1} R^{\mathbb{I}}\right)^{-1} \\
& {\left[\mathrm{r}-\mathrm{R}\left\{\theta+\left(\mathrm{F}^{\mathbb{I}} \Psi^{-1} \mathrm{~F}\right)^{-1}\left(\mathrm{~F}^{\mathbb{I}} \Psi^{-1} \omega\right)\right\}\right],} \\
& \rightarrow \theta^{\mathrm{C}}-\theta=\left(\mathrm{F}^{\mathbb{I}} \Psi^{-1} \mathrm{~F}\right)^{-1}\left(\mathrm{~F}^{\mathbb{I}} \Psi^{-1} \omega\right)-\left(\mathrm{F}^{\mathbb{I}} \mathrm{F}\right)^{-1} R^{\mathbb{I}}\left(R\left(F^{\mathbb{I}} \mathrm{F}\right)^{-1} R^{\mathbb{I}}\right)^{-1} \\
& \mathbb{R}\left(F^{\mathbb{I}} \Psi^{-1} F\right)^{-1}\left(F^{\mathbb{I}} \Psi^{-1} \omega\right) \text {. } \\
& \rightarrow \theta^{\mathrm{C}}-\theta=\left(\mathrm{F}^{\mathbb{I}} \Psi^{-1} \mathrm{~F}\right)^{-1} F^{\mathbb{I}} \Psi^{-1} \omega\left(F^{\mathbb{I}} F\right)^{-1} R^{\mathbb{I}}\left[R\left(F^{\mathbb{I}} F\right)^{-1} R^{\mathbb{I}}\right]^{-1}\left(F^{\mathbb{I}} \Psi^{-1} F\right) F^{\mathbb{I}} \Psi^{-1} \omega \\
& =\left[I-\left(F^{I} F\right)^{-1} R^{I}\left(R\left(F^{I} F\right)^{-1} R^{I}\right)^{-1} R\right]\left(F^{I} \Psi^{-1} F\right)^{-1}\left(F^{I} \Psi^{-1} \omega\right) \\
& =\mathbb{M}^{\mathrm{C}}\left(\mathrm{F}^{\mathbb{I}} \Psi^{-1} \mathrm{~F}\right)^{-1}\left(\mathrm{~F}^{\mathrm{I}} \Psi^{-1}+\omega\right)
\end{aligned}
$$

where $M^{\mathbb{C}}=\left[\mathrm{I}-\left(\mathrm{F}^{\mathbb{I}} \mathrm{F}\right)^{-1} \mathrm{R}^{\mathbb{I}}\left(\mathrm{R}\left(\mathrm{F}^{\mathrm{I}} \mathrm{F}\right)^{-1} \mathrm{R}^{\mathbb{I}}\right)^{-1} \mathrm{R}\right]$ and $\omega=Y-F_{z}$

$\therefore \theta^{\mathrm{c}}-\theta=\mathrm{M}^{\mathrm{c}}(\hat{\theta}-\theta)$.

Lemma1: the matrix $M^{\mathrm{C}}=\left[\mathrm{I}-\left(\mathrm{F}^{\mathrm{I}} \mathrm{F}\right)^{-1} \mathrm{R}^{\mathrm{I}}\left(\mathrm{R}\left(\mathrm{F}^{\mathrm{I}} \mathrm{F}\right)^{-1} \mathrm{R}^{\mathrm{I}}\right)^{-1} \mathrm{R}\right]$ is idempotent

Proof: $M^{\mathrm{C}} M^{\mathrm{C}}=\left[\mathrm{I}-\left(\mathrm{F}^{\mathrm{I}} \mathrm{F}\right)^{-1} \mathrm{R}^{\mathrm{I}}\left(\mathrm{R}\left(\mathrm{F}^{\mathrm{I}} \mathrm{F}\right)^{-1} \mathrm{R}^{\mathrm{I}}\right)^{-1} \mathrm{R}\right]\left[\mathrm{I}-\left(\mathrm{F}^{\mathrm{I}} \mathrm{F}\right)^{-1} \mathrm{R}^{\mathrm{I}}\left(\mathrm{R}\left(\mathrm{F}^{\mathrm{I}} \mathrm{F}\right)^{-1} R^{\mathrm{I}}\right)^{-1} \mathrm{R}\right]$

$$
\begin{aligned}
= & I-\left(F^{\mathbb{I}} F\right)^{-1} R^{\mathbb{I}}\left(R\left(F^{\mathbb{I}} F\right)^{-1} R^{\mathbb{I}}\right)^{-1} R-\left(F^{\mathbb{I}} F\right)^{-1} R^{\mathbb{I}}\left(R\left(F^{\mathbb{I}} F\right)^{-1} R^{\mathbb{I}}\right)^{-1} R \\
& +\left(F^{\mathbb{I}} F\right)^{-1} R^{\mathbb{I}}\left(R\left(F^{\mathbb{I}} F\right)^{-1} R^{\mathbb{I}}\right)^{-1} R\left(F^{\mathbb{I}} F\right)^{-1} R^{\mathbb{I}}\left(R\left(F^{\mathbb{I}} F\right)^{-1} R^{\mathbb{I}}\right)^{-1} R \\
= & I-2\left(F^{\mathbb{I}} F\right)^{-1} R^{\mathbb{I}}\left(R\left(F^{\mathbb{I}} F\right)^{-1} R^{\mathbb{I}}\right)^{-1} R+\left(F^{\mathbb{I}}\right)^{-1} R^{\mathbb{I}}\left(R\left(F^{\mathbb{I}} F\right)^{-1} R^{\mathbb{I}}\right)^{-1} R \\
= & I-\left(F^{\mathbb{I}} F\right)^{-1} R^{\mathbb{I}}\left(R\left(F^{\mathbb{I}} F\right)^{-1} R^{\mathbb{I}}\right)^{-1} R .
\end{aligned}
$$

Theorem1: suppose we have the random panel data model then the constrained maximum likelihood estimator $\theta^{c}$ is unbiased estimator of $\theta$ when the constraint is true and the variance of $\theta^{\mathrm{C}}$ is $\mathrm{M}^{\mathrm{C}}\left(\mathrm{F}^{\mathrm{I}} \Psi^{-1} \mathrm{~F}\right)^{-1}$.

Proof: Since $\theta^{e}=\hat{\theta}+\left(F^{T} F\right)^{-1} R^{T}\left(R\left(F^{T} F\right)^{-1} R^{T}\right)^{-1}(r-R \hat{\theta})$,

$$
\begin{aligned}
\rightarrow E\left(\theta^{c}\right) & =\mathbb{E}(\hat{\theta})+E\left[\left(F^{T} F\right)^{-1} R^{T}\left(R\left(F^{T} F\right)^{-1} R^{T}\right)^{-1}(r-R \hat{\theta})\right\} \\
& =\theta+\left(F^{T} F\right)^{-1} R^{T}\left(R\left(F^{T} F\right)^{-1} R^{T}\right)^{-1}(r-R E(\hat{\theta})) \\
& =\theta+\left(F^{\mathbb{I}} F\right)^{-1} R^{\mathbb{T}}\left(R\left(F^{\mathbb{I}} F\right)^{-1} R^{1}\right)^{-1}(r-R \theta) \\
& =\theta .
\end{aligned}
$$

Now, the variance of $\theta^{C}$ is

$$
\begin{aligned}
& \operatorname{Var}\left(\theta^{\mathrm{C}}\right)=\mathrm{E}\left[\left(\theta^{\mathrm{C}}-\theta\right)\left(\theta^{\mathrm{C}}-\theta\right)^{\mathrm{I}}\right]
\end{aligned}
$$

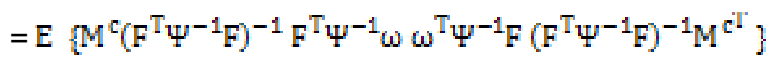

$$
\begin{aligned}
& =M^{\mathrm{C}}\left(\mathrm{F}^{\mathrm{I}} \Psi^{-1} F\right)^{-1} F^{\mathrm{I}} \Psi^{-1} \mathrm{E}\left(\omega^{\mathrm{T}}\right) \Psi^{-1} F\left(F^{\mathrm{T}} \Psi^{-1} F\right)^{-1} M^{\mathrm{C}^{\mathrm{I}}} \\
& =\mathbb{M}^{\mathrm{C}}\left(\mathrm{F}^{\mathrm{T}} \Psi^{-1} \mathrm{~F}\right)^{-1}\left(\mathrm{~F}^{\mathrm{T}} \Psi^{-1} \Psi \Psi^{-1} F\right)\left(\mathrm{F}^{\mathrm{T}} \Psi^{-1} F\right)^{-1} \mathrm{M}^{\mathrm{C}^{\mathrm{I}}} \\
& =M^{\mathrm{C}}\left(\mathrm{F}^{\mathrm{T}} \Psi^{-1} \mathrm{~F}\right)^{-1}\left(\mathrm{~F}^{\mathrm{T}} \Psi^{-1} \mathrm{~F}\right)\left(\mathrm{F}^{\mathrm{T}} \Psi^{-1} \mathrm{~F}\right)^{-1} \mathrm{M}^{\mathrm{C}} \\
& =\mathrm{M}^{\mathrm{C}}\left(\mathrm{F}^{\mathrm{T}} \Psi^{-1} \mathrm{~F}\right)^{-1} \mathrm{M}^{\mathrm{C}^{\mathrm{II}}} \\
& =\left[I-\left(F^{\mathbb{I}} F\right)^{-1} R^{I}\left(R\left(F^{I} F\right)^{-1} R^{I}\right)^{-1} R\right]\left(F^{I} \Psi^{-1} F\right)^{-1} \\
& {\left[\mathrm{I}-\left(\mathrm{F}^{\mathrm{I}} \mathrm{F}\right)^{-1} \mathrm{R}^{\mathrm{I}}\left(\mathrm{R}\left(\mathrm{F}^{\mathrm{I}} \mathrm{F}\right)^{-1} \mathrm{R}^{\mathrm{I}}\right)^{-1} \mathrm{R}\right]} \\
& =\left(F^{\mathbb{I}} \Psi^{-1} F\right)^{-1}-\left(F^{\mathbb{I}} F\right)^{-1} R^{\mathbb{I}}\left(R\left(F^{\mathbb{I}} F\right)^{-1} R^{\mathbb{I}}\right)^{-1} R\left(F^{\mathbb{I}} \Psi^{-1} F\right)^{-1}
\end{aligned}
$$




$$
\begin{aligned}
& -\left(F^{\mathbb{I}} F\right)^{-1} R^{\mathbb{I}}\left(R\left(F^{\mathbb{I}} F\right)^{-1} R^{\mathbb{I}}\right)^{-1} R\left(F^{\mathbb{I}} \Psi^{-1} F\right)^{-1}+\left(F^{\mathbb{I}} F\right)^{-1} R^{\mathbb{I}}\left(R\left(F^{\mathbb{I}} F\right)^{-1} R^{\mathbb{I}}\right)^{-1} \\
& R\left(F^{\mathbb{I}} \Psi^{-1} F\right)^{-1}\left(F^{\mathbb{I}} F\right)^{-1} R^{\mathbb{I}}\left(R\left(F^{\mathbb{I}} F\right)^{-1} R^{\mathbb{I}}\right)^{-1} R \\
= & \left(F^{\mathbb{I}} \Psi^{-1} F\right)^{-1}-2\left(F^{\mathbb{I}} \Psi^{-1} F\right)^{-1} R^{\mathbb{I}}\left(R\left(F^{\mathbb{I}} \Psi^{-1} F\right)^{-1} R^{\mathbb{I}}\right)^{-1} R\left(F^{\mathbb{I}} \Psi^{-1} F\right)^{-1} \\
& +\left(F^{\mathbb{I}} \Psi^{-1} F\right)^{-1} R^{\mathbb{I}}\left(R\left(F^{\mathbb{I}} \Psi^{-1} F\right)^{-1} R^{\mathbb{I}}\right)^{-1} R \\
& =\left(F^{\mathbb{I}} \Psi^{-1} F\right)^{-1}-\left(F^{\mathbb{I}} \Psi^{-1} F\right)^{-1} R^{\mathbb{I}}\left(R\left(F^{\mathbb{I}} \Psi^{-1} F\right)^{-1} R^{\mathbb{I}}\right)^{-1} \\
= & {\left[I-R^{\mathbb{I}}\left(R\left(F^{\mathbb{I}} \Psi^{-1} F\right)^{-1} R^{\mathbb{I}}\right)^{-1}\right]\left(F^{\mathbb{I}} \Psi^{-1} F\right)^{-1} } \\
= & M^{\mathbb{C}}\left(F^{\mathbb{I}} \Psi^{-1} F\right)^{-1} .
\end{aligned}
$$

\section{Theorem2:}

consider the random panel data model (5) the likelihood ratio test for $H_{0^{x}}$ random panel data model against

$H_{1}$ restricted random panel data model is

$$
\frac{\frac{\left(Y-F \theta^{c}\right)^{T}\left(Y-F \theta^{c}\right)-(Y-F \hat{\theta})^{\mathbb{T}}(Y-F \hat{\theta})}{m}}{\frac{m}{N-F \cdot)^{\mathrm{T}}(Y-F \hat{\theta})}} \sim F\left(m{ }_{*} N T-K\right)
$$

\section{Proof:}

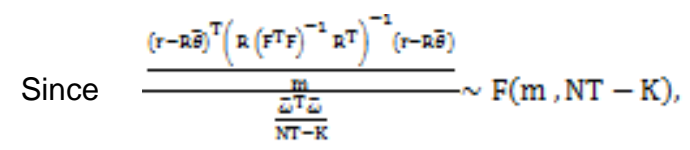

using (25) in (32) to obtain

$$
\frac{\frac{\omega^{\mathrm{c}} \omega^{\mathrm{c}}-\hat{\omega}^{T} \hat{\omega}}{\mathrm{m}}}{\frac{\widehat{\omega}^{\mathrm{T}} \hat{\omega}}{\mathrm{NT}-\mathrm{K}}} \sim \mathrm{F}\left(\mathrm{m}_{\mathrm{n}} \mathrm{NT}-\mathrm{K}\right)
$$

From (16) we can find the restricted likelihood function at the restricted maximum likelihood estimators as follows

$L^{*}=(2 \pi)^{-\frac{\mathbb{N} T}{2}}\left[\frac{1}{N}\left(Y-F \theta^{C}\right)^{T} P\left(Y-F \theta^{C}\right)\right] e^{-\frac{\mathbb{N}}{2}}\left[\frac{1}{\mathbb{N}(T-1)}\left(Y-F \theta^{C}\right)^{T} Q\left(Y-F \theta^{C}\right) e^{-\frac{N(T-2)}{2}}\right.$.

and from (6) we can find the likelihood function at the maximum likelihood estimators as follows

$L=(2 \pi)^{-\frac{\mathbb{N}}{2}}\left[\frac{1}{\mathbb{N}}(Y-F \hat{\theta})^{T} P(Y-F \hat{\theta})\right] e^{-\frac{\mathbb{N}}{2}}\left[\frac{1}{N(T-1)}(Y-F \hat{\theta})^{T} Q(Y-F \hat{\theta})\right] e^{-\frac{N(T-2)}{2}}$.

Then, the likelihood ratio statistical is

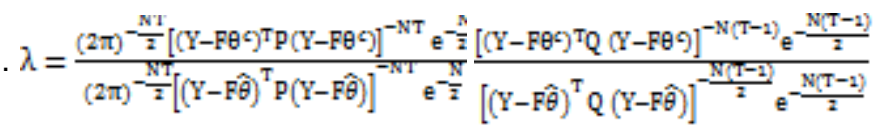

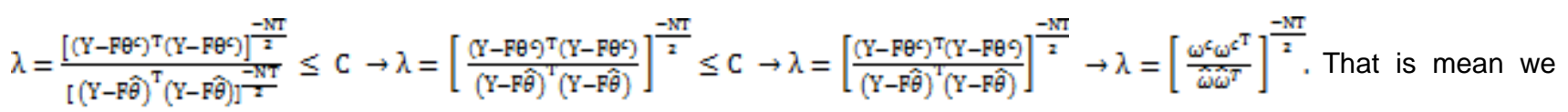

$$
\begin{aligned}
& \text { reject the null hypothesis if }
\end{aligned}
$$

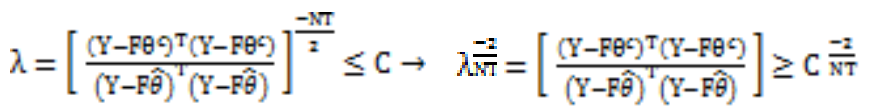

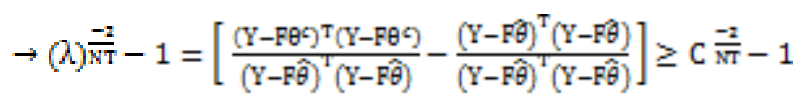

$$
\begin{aligned}
& \rightarrow(a) \frac{-z}{\mathrm{NT}}-1=\frac{\left(\mathrm{Y}-\mathrm{F \theta} \cdot \mathrm{T}(\mathrm{Y}-\mathrm{F \theta})-(\mathrm{Y}-\mathrm{F} \hat{\theta})^{\mathrm{T}}(\mathrm{Y}-\mathrm{F} \theta)\right.}{(\mathrm{Y}-\mathrm{F} \hat{\theta})} \geq \mathrm{C} \frac{-\mathrm{z}}{\mathrm{NT}}-1
\end{aligned}
$$

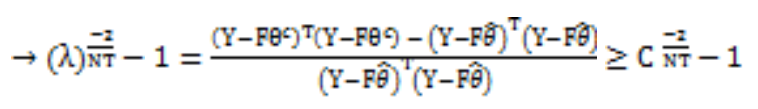




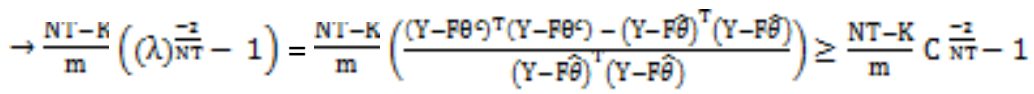

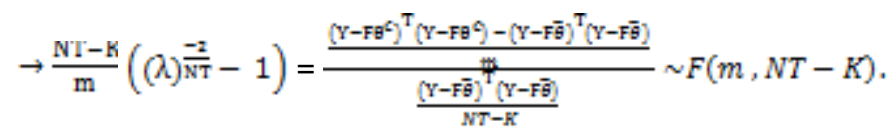

\section{SECTIONS}

The conclusions which are obtained throughout this paper are given as follows :

1-The maximum likelihood estimators of parameters $\theta, \sigma_{\mathbb{g}}^{2}, \sigma_{1}^{2}$ of panel data model are

$$
\hat{\theta}=\left(F^{T} \Psi^{-1} F\right)^{-1}\left(F \Psi^{-1} Y\right), \hat{\sigma}_{z}^{2}=\frac{1}{\mathbb{N}(\mathbb{T}-1)}(Y-F \hat{\theta})^{\mathbb{T}} Q(Y-F \hat{\theta}) \quad \text { and } \quad \tilde{\sigma}_{1}^{2}=\frac{1}{\mathbb{N}}(Y-F \hat{\theta})^{1} P(Y-F \hat{\theta})
$$

2-The restricted maximum likelihood estimator of parameters $\theta^{\mathrm{C}}{ }_{\mathrm{x}} \sigma_{\mathrm{g}}^{2} \sigma_{1}^{2}$ are

$$
\begin{aligned}
& \theta^{\mathrm{C}}=\hat{\theta}+\left(F^{\mathrm{T}} \mathrm{F}\right)^{-1} R^{\mathrm{T}}\left(\mathrm{R}\left(\mathrm{F}^{\mathrm{T}} \mathrm{F}\right)^{-1} \mathrm{R}^{\mathrm{T}}\right)^{-1}(\mathrm{r}-\mathrm{R} \hat{\theta}), \\
& \hat{\sigma}_{\mathrm{g}}^{2}=\frac{1}{\mathrm{~N}(\mathrm{~T}-1)}\left(\mathrm{Y}-\mathrm{F} \theta^{\mathrm{C}}\right)^{\mathrm{T}} \mathrm{Q}\left(\mathrm{Y}-\mathrm{F} \theta^{\mathrm{C}}\right) \text { and } \hat{\sigma}_{1}^{2}=\frac{1}{\mathrm{~N}}\left(\mathrm{Y}-\mathrm{F} \theta^{\mathrm{C}}\right)^{\mathrm{T}} \mathrm{P}\left(\mathrm{Y}-\mathrm{F} \theta^{\mathrm{C}}\right)
\end{aligned}
$$

3-The constrained maximum likelihood estimator $\theta^{C}$ is unbiased estimator of $\theta$ when the constraint is true $s$

4-The likelihood ratio test for $H_{0^{x}}$ random panel data model against $H_{1^{x}}$ restricted random panel data model is

$$
\frac{\frac{\left(\mathrm{Y}-\mathrm{F} \theta^{\mathrm{C}}\right)^{\mathrm{T}}\left(\mathrm{Y}-\mathrm{F} \theta^{\mathrm{C}}\right)-(\mathrm{Y}-\mathrm{F} \hat{\theta})^{\mathrm{T}}(\mathrm{Y}-\mathrm{F} \hat{\theta})}{m}}{\frac{m}{N-\mathrm{F})^{\mathrm{T}}(\mathrm{Y}-\mathrm{F} \tilde{\theta})}} \sim F(m, N T-K) .
$$

\section{REFERENCES}

[1] Ashley ,Richard A, Sun , Xiaojin," subset continuous updating GMM estimators for dynamic panel data model, Econometrics,4,47; doi:10.3390/econometrics 4040047, (2016).

[2] Baltagi , badi, " Econometric Analysis of panel data “, John Wily \& Sons Inc. third edition , (2005).

[3] Bun ,Maurice J.G. and Carree, Martin A., "Bias corrected estimation in dynamic panel data model with heteroscedasticity ",UVA Econometrics, (2005).

[4] Elhorst,J. Paul, "panel data models extended to spatial error autocorrelation or spatially lagged dependent variable "university of Groningen(2001).

[5] Feng ,Gao, Jiti , Peng Bin and Zhang ,Xiaohui ," a varying coefficient panel data model with fixed effects ",ISSN 1440-771X, Monash University,(2015).

[6] Frees , Edward W. "Longitudinal and Panel Data: Analysis and Applications for the Social Sciences" This draft is partially funded by the Fortis Health Insurance Professorship of Actuarial Science. Cambridge University Press (2004) .

[7] Gorgens ,Tue, Skeels, Christopher L. and Wurtz , Allan H., "efficient estimation of nonlinear dynamic panel data models with application to smooth transition models",(2008).'

[8] Hurlin ,Christophe, " testing granger causality in heterogeneous panel data model with fixed coefficients " , university Paris, (2004).

[9] Husio, Cheng, " Analysis of panel data “ ,second edition, Cambridge university Press, (2003).

[10] Lewis, John R.," Bayesian Restricted Likelihood Method " ,Technical Report No. 878,(2014).

[11] Rao, C. Rad hakrishna and Toutenburg,Helge,"Linear Models Least Squares and Alternatives", Second edition, springer,(1999).

[12] Rencher, Alvin C. and Schaalje, G. Bruce, " Linear Models in Statistics " ,second edition, Wiley-Interscience, A John Wiley \& Sons ,Inc., Publication,(2008).

[13] Sun, Jianguo \& Zhao, Xingqiu , "Statistical Analysis of Panel Count Data “, Springer ,

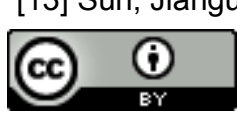

This work is licensed under a Creative Commons Attribution 4.0 International License. 